\title{
Session V
}

Coronal Heating and Solar Wind Acceleration

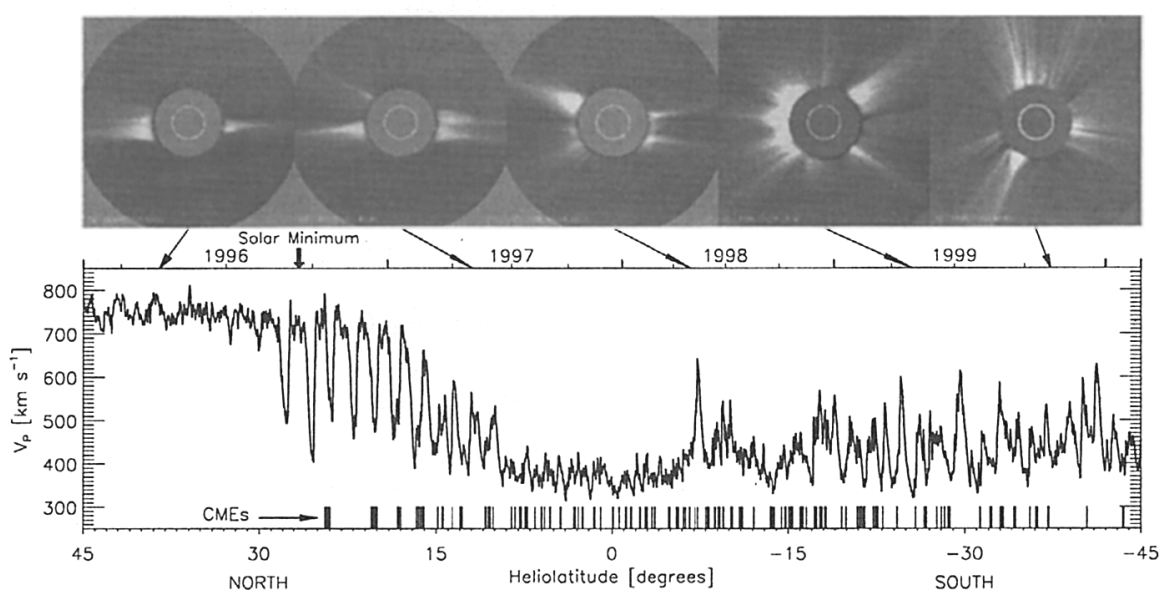

The changing solar corona as seen by the coronagraph on SOHO and the solar wind as observed by Ulysses. The solar wind speed is plotted versus heliolatitude, covering a range of $\pm 45^{\circ}$, corresponding to the time period from the past solar activity minimum in 1996 to the present year 2000 (adapted from Marsch, p. 447) 


\title{
Observations and Models of the Fast and Slow Solar Wind
}

\author{
E. Marsch
}

\author{
Max-Planck-Institut für Aeronomie, 37191 Katlenburg-Lindau, Germany
}

\begin{abstract}
There are two major types of solar wind. The steady fast wind originating on open magnetic field lines in coronal holes, and the unsteady slow wind coming from the bulk or boundary layer of streamers, which are mostly magnetically closed but may open up temporarily. Many observations of the solar wind have in the past been made in situ and remotely. Key empirical constraints, which are imposed on the models by the Helios (near-Sun, in-ecliptic) and Ulysses (high-latitude) interplanetary measurements and by the Solar and Heliospheric Observatory (SOHO) plasma diagnostics, are discussed with respect to the fluid and kinetic properties of the wind. The majority of the models is concerned with the fast wind, essential properties of which can be reproduced by 1-D multi-fluid models involving broad-band waves. Owing to its timevariable nature, no robust understanding of the slow wind exists. Selected results from modelling and observations are discussed.
\end{abstract}

\section{Large-scale solar corona and solar wind}

From Helios, Ulysses and the Solar and Heliospheric Observatory (SOHO) it has become abundantly clear that two characteristic types of solar corona and solar wind exist, prevailing at different heliographic latitudes and longitudes and phases of the solar cycle. The magnetic field and plasma flow pattern is most simple and conspicuous near solar minimum, when fast wind dominates and emanates steadily from the magnetically open polar coronal holes (Woch et al. 1997). In contrast, the slow wind is unsteady and originates abruptly from the equatorial streamer belt, which may open intermittently and release plasma blobs (Sheeley et al. 1997) and coronal mass ejections (CMEs), or more continuously from the boundary layers embedding multiple streamers, which may merge and form the heliospheric current sheet. In-situ measurements and coronagraph images indicate that during solar minimum the transitions from fast to slow flows are rather sharp, with the slow flows being restricted to within about $\pm 20^{\circ}$ around the equator of the Sun.

The large-scale structure of the coronal magnetic field changes over the 11-year solar cycle. The field becomes particularly simple in the minimumactivity period, where it can be described essentially by a dipolar and quadrupolar component supplemented by contributions from a near-Sun current sheet. Banaszkiewicz et al. (1997) provided a simple algebraic model for the solar magnetic field near minimum. The model describes the global field (with the radial component denoted here by $B(r)$ ) and also allows one to derive the ex- 


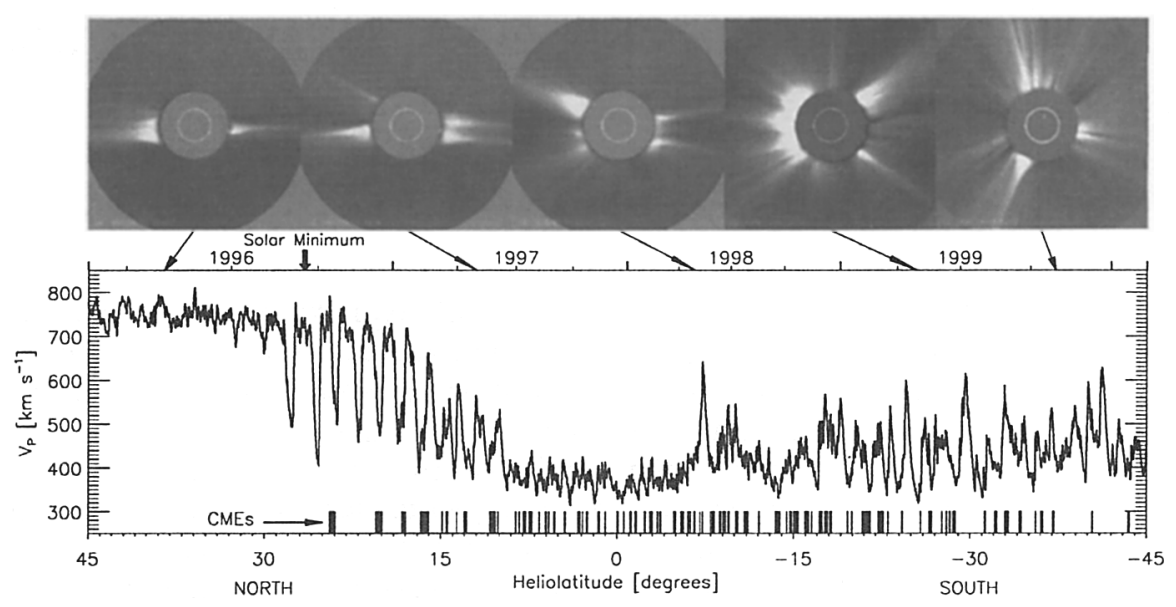

Figure 1. The changing solar corona as seen by the coronagraph on SOHO and the solar wind as observed by Ulysses. The solar wind speed is plotted versus heliolatitude, covering a range of $\pm 45^{\circ}$, corresponding to the time period from the past solar activity minimum in 1996 to the present year 2000 .

pansion factor, $A(r)$, of magnetic flux tubes with distance, $r$, from the Sun. The area function is required in 1-D models of the fast solar wind originating in the polar coronal holes $(\mathrm{CHs})$.

The changing solar corona, as seen by the coronagraph on SOHO and the solar wind as observed by Ulysses, is shown as a function of heliolatitude in Figure 1 (courtesy D. McComas). While approaching solar maximum, the corona develops an increasingly complex 3-D structure with a correspondingly variable solar wind flow pattern. No fast wind solar wind is observed any more at higher latitudes, in contrast to the near-minimum period with its extended fast streams over the polar regions. Note the overall steadiness of the fast wind and the variability of the slow wind, intermingled with fast recurrent streams from low-latitude coronal holes during minimum. The slow wind prevails near solar maximum. Figure 1 provides a concise overview of the three-dimensional heliosphere by means of the solar wind speed measured by Ulysses. Many of the important results obtained from the Ulysses mission are described in the articles of the book edited by Marsden (1995).

A study of the heliospheric magnetic field as a function of distance, longitude and latitude in the inner heliosphere was made by Forsyth (1995) and Whang (1995), showing that the distant radial field was rather monopolar, with large compressive deviations occurring near the heliospheric current sheet. During the solar activity cycle the current sheet changes considerably. It becomes most complicated, highly structured and warped around the activity maximum, because the Sun then has a complex multipole field and many differently sized 
activity regions. For the recent epoch see the article of Bravo et al. (1998) on the changing solar magnetic field. Traditionally, Hoeksema (1995) has monitored the associated evolution of the current sheet and described it by means of plots of the source field polarity at $2.5 R_{\odot}$, as reconstructed from potential field extrapolations of the solar surface fields.

\section{Coronal temperatures and boundary conditions}

Here we will concentrate on the properties of the fast solar wind which is characterised by the following parameters: Total energy flux at $1 R_{\odot}: F_{E}=510^{5}$ erg $\mathrm{cm}^{-2} \mathrm{~s}^{-1}$; speed beyond $10 R_{\odot}: V_{p}=(700-800) \mathrm{km} \mathrm{s}^{-1}$; at 1 AU, typical proton flux: $n_{p} V_{p}=210^{8} \mathrm{~cm}^{-2} \mathrm{~s}^{-1}$, density: $n_{p}=3 \mathrm{~cm}^{-3}$, helium abundance: $n_{\alpha} / n_{p}=0.04$, and average temperatures: $T_{p}=310^{5} \mathrm{~K}, T_{\alpha}=10^{6} \mathrm{~K}$, and $T_{e}=1.510^{5} \mathrm{~K}$. For the heavy ions one finds that $T_{i} \approx m_{i} / m_{p} T_{p}$ and $\mathbf{V}_{i}-\mathbf{V}_{p}=\mathbf{V}_{A}$, indicating the effects of wave-particle intercations. All these parameters have to be met by any solar wind model.

The electron density and temperature in the corona are of paramount importance, since they not only characterize the collisional properties and the electron pressure in the corona, but also determine the ionization state of hydrogen, helium and heavier elements. Electrons govern the radiative losses in the EUV and soft $\mathrm{X}$ rays through collisional line excitation (see e.g. Mariska's book, 1992). Strictly speaking it is the detailed electron distribution function that matters, which is presently incapable of measurement close to the Sun, yet well known farther away, where nonthermal features prevail, such as the suprathermal strahl and the core-halo structure (Pilipp et al. 1987).

Line ratio techniques have been used to determine the electron density and to establish with SOHO the electron density profiles for the first few 100 arcsec above the limb in various regions. E.g., Wilhelm et al. (1998) have used the Si VIII $1445.73 \AA / 1440.51 \AA$ line ratio, yielding a density that starts at $10^{8} \mathrm{~cm}^{-3}$ at $20 \operatorname{arcsec}(1 \operatorname{arcsec} \approx 715 \mathrm{~km})$ and drops to about $610^{7} \mathrm{~cm}^{-3}$ at an altitude of 300 arcsec in the polar $\mathrm{CH}$. This profile smoothly continues into the radial profile of $n_{e}$ as estimated by the measurements of oxygen line intensities (Corti et al. 1997). Given these density profiles, and the complementing ones obtained from coronagraphs, one can infer a corresponding empirical flow speed of the plasma by exploiting the mass continuity equation and assuming a flow or flux tube geometry. This technique has been applied by Fisher \& Guhathakurta (1995). Their results imply strong acceleration close to the Sun.

Barnes et al. (1995) showed clearly that during solar minimum activity the mass flux and helium abundance of the fast solar wind are remarkably stable and constant all over the solar surface with the exception of the equatorial streamer belt, where transients and CMEs render the mass flux more varibale. They quoted a mean value of the proton flux density of $2.210^{8} \mathrm{~cm}^{-2} \mathrm{~s}^{-1}$ normalized to $1 \mathrm{AU}$, consistent with the older Helios measurements two solar cycles earlier.

One of the major surprises of $\mathrm{SOHO}$ was that electrons were rather cold in $\mathrm{CHs}$, evidence that came from the $T_{e}$ estimates obtained from EUV emission lines. Wilhelm et al. (1998) used the Mg IX 749.55 $\AA$ / 706.07 $\AA$ line ratio and concluded that $T_{e}$ hardly ever reaches the canonical value of $1 \mathrm{MK}$, but remains below and rapidly falls off with height in the CH. Similarly, David et al. (1998) 


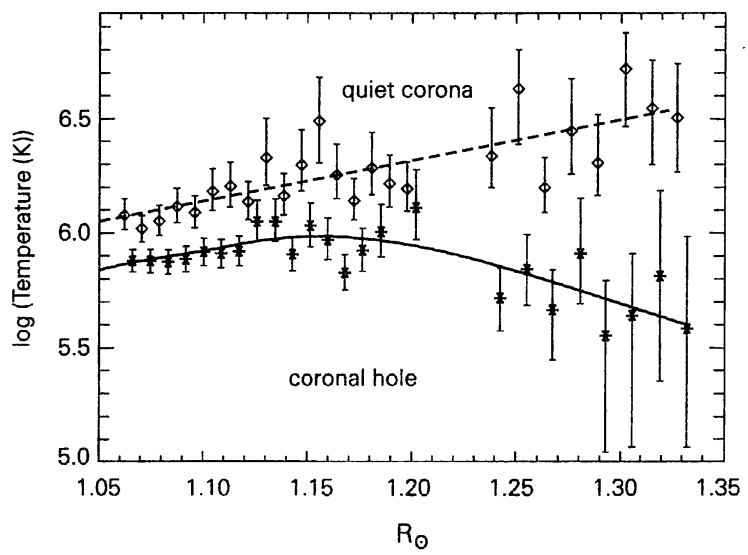

Figure 2. The electron temperature profiles in the corona as obtained from EUV line-ratio diagnostics with the spectrometers on SOHO are shown as a function of distance from the Sun (from David et al. 1998). Note the low temperature in the polar coronal hole and the comparatively high temperature in the equatorial streamer belt.

derived, in a synergistic effort of the SOHO spectrometers using the line pair O vi $173 \AA / 1032 \AA$, the electron temperature profiles in an equatorial streamer at the west limb and a coronal hole at the polar north limb. Their data shown in Figure 2 demonstrate that electrons cool off rapidly and only have a $T_{e}$ of about $0.5 \mathrm{MK}$ at $1.3 R_{\odot}$. Such low electron temperatures are consistent with the Helios interplanetary in-situ measurements, which clearly indicated that in the fast flows electrons are cold and their radial temperature gradients flat (see the review by Marsch 1991).

Concerning the solar corona, SOHO brought also new insights into the temperature distributions of the ions in the solar atmosphere and provided clear evidence for deviations from collisional equilibrium. Seely et al. (1997) showed that the temperatures of minor ions are generally quite high, at a few MK, and exceed clearly the electron temperature. Cranmer et al. (1999a) found the temperature of oxygen $\mathrm{O}^{5+}$ in a coronal hole at around $3 R_{\odot}$ to be as high as $100 \mathrm{MK}$. Tu et al. (1998) investigated the temperatures of ions in the polar coronal holes below $1.3 R_{\odot}$ and found high values of $T_{i}$, ranging from 2 to $6 \mathrm{MK}$. The different ion temperatures were analysed with respect to the ionic mass-per-charge, $m_{i} / e_{i}$, values and compared, discriminating them by their fundamental atomic parameters without assumptions on the local plasma state in the emission region. Evidence was found that the heating of the heavy ions is caused by cyclotron-resonance with high-frequency Alfvén waves. Similar strong evidence for cyclotron-resonant heating of ions was earlier found in situ in the fast solar wind (see again the review by Marsch 1991).

The Lyman series of hydrogen was observed with SOHO on the north polar limb of the Sun with a total exposure time of more than ten hours. The resulting 
line profiles have been analysed by Marsch et al. (2000) in order to infer the proton temperature in the transition region at the coronal base. The hydrogen temperature gradient was found to be unexpectedly small and did nor reveal as steep a jump as expected from conventional modelling of the transition region (Mariska 1992). The average temperature increased slightly from 1 to $210^{5} \mathrm{~K}$ in the height range from 12000 to $18000 \mathrm{~km}$.

\section{Multi-fluid equations involving wave dissipation}

The new SOHO observations indicate substantial deviations of the coronal plasma from local collisional or thermal equilibrium. Therefore, the models must use the multi-fluid equations in order to account for this fact and describe adequately the thermodynamics and internal energy state of the coronal and solar wind plasma. Various forms of the fluid equations have been used in the many papers attempting to model the solar wind. Here we only quote the stationary equations of motion for any particle species $j$ and a special 1-D geometry described by the flow(flux)-tube area function $A(r)$, where the distance from the Sun is denoted by $r$. Conservation of magnetic flux implies that $B(r) A(r)$ be constant. The mass continuity equation reads

$$
\frac{d}{d r}\left(A(r) \rho_{j} U_{j \|}\right)=0
$$

In the momentum equation, gravity and the electric field, $E_{\|}$, stemming from the electron pressure gradient, need also be considered. Since the observations of SOHO and Helios indicate that $T_{e} \leq T_{p} \leq T_{j}$, the interplanetary electric field is usually much smaller than the ion partial-pressure gradients. For a 1-D radial field-line geometry we have the following set:

$$
\left(1-\frac{V_{j \|}^{2}}{U_{j \|}^{2}}\right) U_{j \|} \frac{d}{d r} U_{j \|}-V_{j \perp}^{2} \frac{d \ln (A(r))}{d r}+\frac{d}{d r} V_{j \|}^{2}+\frac{G M_{\odot}}{r^{2}}-\frac{e_{j}}{m_{j}} E_{\|}=\frac{\partial}{\partial t} U_{j \|}+a_{j \|} .
$$

The two energy equations, put here in terms of the perpendicular and parallel thermal speeds squared, are given by

$$
\begin{gathered}
U_{j \|}\left(\frac{d}{d r} V_{j \perp}^{2}+V_{j \perp}^{2} \frac{1}{A(r)} \frac{d A(r)}{d r}\right)=\frac{\partial}{\partial t} V_{j \perp}^{2}+Q_{j \perp}+S_{j \perp}, \\
U_{j \|} \frac{d}{d r} V_{j \|}^{2}+2 V_{j \|}^{2} \frac{d}{d r} U_{j \|}=\frac{\partial}{\partial t} V_{j \|}^{2}+Q_{j \|}+S_{j \|} .
\end{gathered}
$$

The big unknowns are the terms on the right-hand sides. They contain the acceleration, $a_{j \|}$, due to Alfvén waves and collisional friction between different species, and furthermore the sources and sinks, $Q_{j \perp, \|}$ and $S_{j \perp, \|}$, of internal or thermal energy, for example due to heat conduction, collisional energy exchange or radiative losses (see, e.g, $\mathrm{Hu}$ et al. 1997, and $\mathrm{Li}$ et al. 1999). In a collisionless medium the most important terms are the ones related to wave-particle interactions. They are generally functionals of the velocity distribution functions (VDF) and/or nonlinear functions of their three basic moments, $U_{j \|}, V_{j \|}$, and 
$V_{j \perp}$, but also functionals of the wave magnetic power spectral density (PSD), $\mathcal{B}_{M}(\mathbf{k})$.

The following definitions hold: The particle's charge is $e_{j}$, its mass $m_{j}$, and density $n_{j}$. The ion gyrofrequency, which by definition carries the sign of the charge, reads as follows: $\Omega_{j}=\left(e_{j} B\right) /\left(m_{j} c\right)$. The mass density of species $j$ is defined as $\rho_{j}=n_{j} m_{j}$, with the total mass density $\rho=\sum_{j} n_{j} m_{j}$. The Alfvén velocity is $V_{A}^{2}=B^{2} /(4 \pi \rho)$. We can then write, after Marsch and Tu (2000), the wave acceleration and heating rates as integrals over the spectrum:

$$
\begin{array}{r}
\left(\begin{array}{c}
\frac{\partial}{\partial t} U_{j \|} \\
\frac{\partial}{\partial t} V_{j \|}^{2} \\
\frac{\partial}{\partial t} V_{j \perp}^{2}
\end{array}\right)=\frac{1}{(2 \pi)^{3}} \int_{-\infty}^{+\infty} d^{3} k \sum_{M} \hat{\mathcal{B}}_{M}(\mathbf{k})\left(\frac{\Omega_{j}}{k}\right)^{2} \frac{1}{1-\left|\hat{\mathbf{k}} \cdot e_{M}(\mathbf{k})\right|^{2}} \\
\times \sum_{s=-\infty}^{+\infty} \mathcal{R}_{j}(\mathbf{k}, s)\left(\begin{array}{c}
k_{\|} \\
2 k_{\|} w_{j}(\mathbf{k}, \mathbf{s}) \\
s \Omega_{j}
\end{array}\right) .
\end{array}
$$

Here we have introduced the spectrum $\hat{\mathcal{B}}_{M}(\mathbf{k})$, which is normalized to the background magnetic field density, $B^{2} / 8 \pi$. The symbol $e_{M}(\mathbf{k})$ denotes the polarization vector of a given wave mode $M$ with frequency $\omega_{M}(\mathbf{k})$ and wave vector $\mathbf{k}$, and $s$ denotes the order of the Bessel function involved in the resonance function or wave absorption coefficient, $\mathcal{R}_{j}(\mathbf{k}, s)$, which is a functional of the VDF and given explicitly in Marsch and Tu (2000). The parallel integration in the velocity-space integrals involved was carried out, whereby we introduced the $s$-order parallel resonant speed in the bulk frame of species $j$ through $w_{j}(\mathbf{k}, s)=\left(\omega_{M}(\mathbf{k})-k_{j \|} U_{j \|}-s \Omega_{j}\right) / k_{\|}$. It plays a key role in the cyclotron or Landau resonance.

The equations $(1-5)$ form a possible set for a complete multi-fluid description of solar wind electrons and ions. Similar sets of equations have been solved numerically by many authors (see the subsequent section). The waveparticle terms can only be evaluated once the VDF, $f_{j}\left(V_{\perp}, V_{\|}\right)$, and the PSD, $\hat{\mathcal{B}}_{M}\left(k_{\perp}, k_{\|}\right)$, are known explicitly. In the solar wind literature, often a simple power-law form of the spectrum was assumed, such as in the early studies by Isenberg \& Hollweg (1983) or recently by Cranmer et al. (1999a, 1999b) for coronal hole heating. However, as Marsch et al. (1982) have previously shown and Tu and Marsch (2000) recently, this approximation is not sufficient, and therefore the full quasi-linear equations should be used to calculate the wave spectrum self-consistently.

\section{Selected model results}

McKenzie et al. (1995) developed a theory for the high-speed solar wind with a simple dissipation length characterization of wave heating of the coronal plasma close to the Sun, with the dissipation length ranging between 0.25 and $0.5 R_{\odot}$. They obtained rapid acceleration with a sonic point at about $2 R_{\odot}$ and a high maximum proton temperature, ranging from 8 to $10 \mathrm{MK}$. Such efficient near-Sun dissipation requires high-frequency Alfvén waves in the frequency domain above $1 \mathrm{~Hz}$. McKenzie et al. (1997) have extended these calculations further to include 


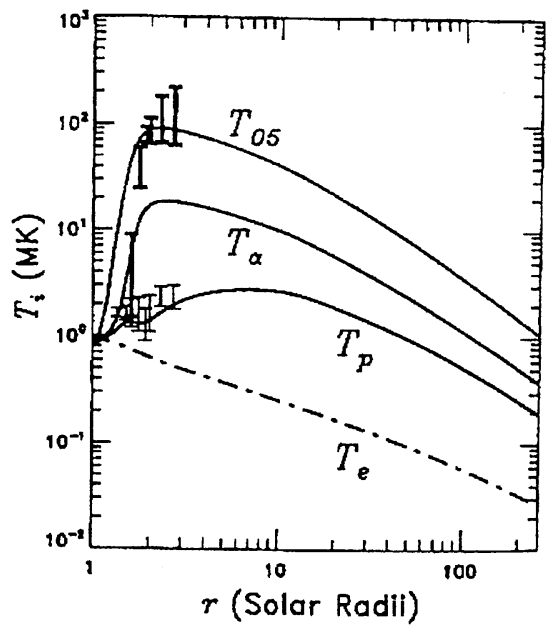

Figure 3. The ion and electron temperature profiles in the solar corona as obtained from a four-fluid model including turbulence-driven heating of heavy ions (after $\mathrm{Hu}$ et al. 2000). Note the sizable temperature differences and the very high oxygen temperature in comparison with the cold electrons. Bars indicate the SOHO measurements.

also the proton temperature anisotropy. Hansteen and Leer (1995) and Esser et al. (1997) have presented parameter studies on the effects of hot protons on the nascent solar wind in the inner corona. A high $T_{p}$ is a genuine property of these models, and thus flow velocities of up to $800 \mathrm{~km} / \mathrm{s}$ are readily obtained within $10 R_{\odot}$ by a deposition of heat or momentum within a fraction of a solar radius above the Sun's surface. Li et al. (1997) studied recently in detail the influence of heavy ions on the fast wind in a three-fluid model. They concluded that preferential heating of heavy ions, in particular of alpha particles, has an impact on the bulk flow which must be considered in wind models.

Detailed parametric studies of the fast solar wind in models including the proton temperature anisotropy have been carried out by $\mathrm{Li}$ et al. (1999) and Hu et al. (1997), who found that anisotropy has little influence on the dynamics yet imposes requirements on the heating mechanism of the solar wind. Modified classical heat-flux laws have been used in models ( $\mathrm{Hu}$ et al. 1997; $\mathrm{Li}$ et al. 1999) for the outer corona and distant wind, mainly because of our lack of understanding of heat conduction in the collisionless solar wind in the presence of strong wave turbulence. The ion and electron temperature profiles in the corona were calculated recently by means of a four-fluid model including turbulencedriven heating of coronal heavy ions after $\mathrm{Hu}$ et al. (2000). Some of their results come close to the observations and are therefore given in Figure 3.

Yet only few of the discussed models have really attempted to describe the detailed physics of the coronal heating mechanism. In an empirical model of a polar coronal hole at solar minimum, Cranmer et al. (1999a,b) analysed the 
spectroscopic constraints placed on the ion cyclotron resonance heating of the high-speed solar wind and investigated the cyclotron wave dissipation caused by heavy minor ions. Furthermore, Tu and Marsch (1997) worked out and corroborated the idea (Axford \& McKenzie 1997), that high-frequency Alfvén wave dissipation may be responsible for the heating, a process well established in the distant solar wind. The key model assumptions are: Proton cyclotron damping of Alfvén waves, running into resonance in a rapidly declining magnetic field, gives fast heating close to the Sun (Marsch et al. 1982); the spectral evolution is slow, and therefore the power spectrum follows the WKB solution. The wave energy is then absorbed by the protons at the local gyrofrequency, i.e. at $\Omega_{p}$. This dissipation builds up a large proton temperature. Input in the model was an unknown power spectrum at the coronal base for which assumption have to be made. Like in the other models the electrons hardly ever reach a $1 \mathrm{MK}$ temperature, whereas the protons attain temperatures of 2-3 MK and effective (including the wave amplitude) temperatures of up to $7 \mathrm{MK}$.

The rapid acceleration obtained in most of the new wave-driven models is achieved by the strong thermal pressure gradient, resulting essentially from the steep ion temperature decline beyond the critical point, beyond which wave absorption weakens or ceases. The wave models can account for both the heating of the corona and acceleration of the solar wind, because it is the high-frequency part of the spectrum which via dissipation directly heats the corona and builds up a strong thermal pressure, and it is the lower-frequency part which pushes additionally on the wind through the wave pressure-gradient force.

\section{Conclusions and questions}

In recent past, considerable progress has been made in characterising the coronal plasma state and in determining the radial profiles of key parameters in the polar coronal holes. Surprisingly, coronal hole ions are generally very hot and electrons cold. These new empirical constraints have to be considered by any theorist modelling the fast solar wind. The slow wind is rather unsteady and comes from the streamer belt near collisional equilibrium. The fast streams are driven by the ion thermal pressure-gradient forces. Certainly, the single-fluid descriptions are outdated and multi-fluid models must be used. In the weakly collisional corona waves and particles are intimately linked through plasma instabilities and wave-particle interactions. They will most likely involve waves at frequencies much higher than considered conventionally in MHD models. There is now clear evidence for heating and acceleration of ions by cyclotron resonance.

Yet, many important questions concerning the corona and wind remain open, such as: What are the processes heating the corona, being either closed with dense plasma in magnetic confinement, or open with dilute plasma escaping freely on magnetic field lines? What determines the solar wind mass flux? Do we really have to invoke complex kinetic physics to understand solar wind accleration? What is the role of MHD and plasma waves and related wave-particle interactions? Is the slow wind a quasi-steady boundary layer flow or genuine, but intrinsically variable, with origin in the transiently opening streamer belt of the Sun? These are important questions still awaiting answers. 


\section{References}

Axford, W.I., \& McKenzie, J.F. 1997, in Cosmic Winds and the Heliosphere, ed. J.R. Jokipii, \& al., (Tucson: The University of Arizona Press), 31

Banaszkiewicz, M., Axford, W.I., \& McKenzie, J.F. 1998, A\&A, 337, 940

Barnes, A., Gazis, P.R., \& Phillips, J.L. 1995, Geophys. Res. Lett., 22, 3309

Bravo, S., Stewart, G.A., \& Blanco-Cano, X. 1998, Solar Phys., 179, 223

Corti, G., Poletto, G., Romoli, M., \& al. 1997, ESA-SP, 404, 289

Cranmer, S.R., Kohl, J.L., Noci, G., \& al. 1999a, ApJ, 511, 481

Cranmer, S.R., Field, G.B., \& Kohl, J.L. 1999b, ApJ, 518, 937

David, C., Gabriel, A.H., Bely-Dubau, F., \& al. 1998, A\&A, 336, L90

Esser, R., Habbal, S.R., Coles, W.A., \& al. 1997, J. Geophys. Res., 102, 7063

Fisher, R., \& Guhathakurta, M. 1995, ApJ, 447, L139

Forsyth, R.J. 1995, Space Science Reviews, 72, 153

Hansteen, V., \& Leer, E. 1995, J. Geophys. Res., 100, 21577

Hoeksema, J.T. 1995, Space Science Reviews, 72, 137

Hu, Y.-Q., Esser, R., \& Habbal, S.R. 1997, J. Geophys. Res., 102, 14661

Hu, Y.-Q., Esser, R., \& Habbal, S.R. 2000, J. Geophys. Res., 105, 5093

Isenberg, P.A., \& Hollweg, J.V. 1983, J. Geophys. Res., 88, 3923

Li, X., Esser, R., \& Habbal, S.R. 1997, J. Geophys. Res., 102, 17419

Li, X., Habbal, S.R., Hollweg, J.V., \& al. 1999, J. Geophys. Res., 104, 2521

McKenzie, J.F., Banaszkiewicz, M., \& Axford, W.I. 1995, A\&A, 303, L45

McKenzie, J.F., Axford, W.I., \& Banaszkiewicz, M. 1997, Geophys. Res. Lett., 24,2877

Mariska, J.T. 1992, The Solar Transition Region, (Cambridge University Press)

Marsch, E. 1991, in Physics of the Inner Heliosphere, Vol. II, ed. R. Schwenn \& E. Marsch, (Heidelberg: Springer Verlag), 45

Marsch, E. \& Tu, C.-Y. 2000, J. Geophys. Res., in press

Marsch, E., Goertz, C. K., \& Richter, K. 1982, J. Geophys. Res., 87, 5030

Marsch, E., Tu, C.-Y., \& Wilhelm, K. 2000, A\&A, 359, 381

Marsden, R.G. (Ed.) 1995, The High Latitude Heliosphere, (Dordrecht: Kluwer Academic Publishers)

Pilipp, W.G., Miggenrieder, H., Montgomery, M.D., \& al. 1987, J. Geophys. Res., 92, 1075

Seely, J.F., Feldman, U., Schühle, U., \& al. 1997, ApJ, 484, L87

Sheeley, N.R.,Jr., Wang, Y.-M., Hawley, S.H., \& al. 1997, ApJ, 484, 472

Tu, C.-Y. \& Marsch, E. 1997, Solar Phys., 171, 363

Tu, C.-Y. \& Marsch, E. 2000, J. Geophys. Res., in press

Tu, C.-Y., Marsch, E., Wilhelm, K., \& Curdt, W. 1998, ApJ, 500, 475

Whang, Y.-M. 1995, Space Science Reviews, 72, 193

Wilhelm, K., Marsch, E., Dwivedi, B.N., \& al. 1998, ApJ, 500, 1023

Woch, J., Axford, W.I., Mall, \& al. 1997, Geophys. Res. Lett., 24, 2885 